\title{
Optical Measurements during Deep Brain Stimulation Lead Implantation: Safety Aspects.
}

Peter Zsigmond, Simone Hemm-Ode and Karin Wårdell

The self-archived postprint version of this journal article is available at Linköping University Institutional Repository (DiVA):

http://urn.kb.se/resolve?urn=urn:nbn:se:liu:diva-144203

N.B.: When citing this work, cite the original publication.

Zsigmond, P., Hemm-Ode, S., Wårdell, K., (2018), Optical Measurements during Deep Brain

Stimulation Lead Implantation: Safety Aspects., Stereotactic and Functional Neurosurgery, 95(6), 392-399. https://doi.org/10.1159/000484944

Original publication available at:

https://doi.org/10.1159/000484944

Copyright: Karger Publishers

http://www.karger.com/ 


\section{Optical measurements during DBS lead implantation - safety aspects}

Peter Zsigmond ${ }^{1}$ MD, PhD, Simone Hemm-Ode ${ }^{2,3}$ MSc, PhD and Karin Wårdell ${ }^{2,4}$ MSc, PhD,

${ }^{1}$ Department of Neurosurgery and Department of Clinical and Experimental Medicine,

Linköping University, Linköping, Sweden

${ }^{2}$ Department of Biomedical Engineering, Linköping University, Sweden

${ }^{3}$ University of Applied Sciences and Arts Northwestern Switzerland

School of Life Sciences, Institute for Medical and Analytical Technologies, Switzerland

${ }^{4}$ Center for Medical Image Science and Visualization, Linköping Sweden

Running title: Optics in DBS surgery

Key words: deep brain stimulation (DBS), laser Doppler flowmetry (LDF), intracerebral hemorrhage ICH), safety

\section{Corresponding author:}

Peter Zsigmond

Department of Neurosurgery

Linköping University Hospital

58185 Linköping

Phone: +46101030000

Email: peter.zsigmond@regionostergotland.se 


\begin{abstract}
Background

Intracerebral hemorrhage (ICH) is the most feared complication in deep brain stimulation (DBS) surgery. The aim of the study was to evaluate patient safety and outcome using laser Doppler flowmetry (LDF) during DBS implantations.
\end{abstract}

\title{
Methods
}

An LDF probe adapted for the stereotactic system was used as guide for creation of the trajectory. The microcirculation along 83 preplanned trajectories was measured during DBS surgery for movement disorders. All positions were investigated from a microcirculatory perspective. Medical record and postoperative radiology were reviewed.

\section{Results}

Of 2963 measurement sites, 234 (7.9\%) showed at least a doubled microvascular blood flow compared to the surrounding tissue. Of these $2.2 \%$ had more than five times higher blood flow in front of the probe tip. Along one trajectory a small ICH was detected during surgery. Increased blood flow was more common close to sulci and verticals.

\section{Conclusion}

Real-time LDF measurement of the microcirculation using a forward looking probe during DBS surgery can detect blood flow peaks and further minimize the risk of developing ICH. No separate guide tube is necessary as the probe also creates the trajectory for the DBS-lead. 


\section{Introduction}

Stereotactic deep brain stimulation (DBS) surgery is since many years used in the treatment of movement disorders and is today a well-recognized technique for reversible neuromodulation. It has been proven during the years to be a very effective therapy in conditions refractory to medical treatment. The most common disorders treated with DBS are Parkinson's disease (PD), essential tremor (ET) and dystonia. Successful surgical treatment with DBS depends on patient selection, accurate targeting and optimal postoperative management, all without adding adverse events. DBS implantation is regarded as a relatively safe neurosurgical procedure but serious adverse events can occur; infections, hardware related problems and intracerebral hemorrhage (ICH). Symptomatic ICH is the most feared serious complication with the highest risk of poor neurological outcome.

Possible risk factors for developing a cerebral hemorrhage can be high age and a history of hypertension [1]. Other risk factors for hemorrhage can be trajectories involving sulci and ventricular penetration [2, 3]. ICH can cause neurologic deficits like hemiparesis, altered consciousness, visual loss and most seriously death. Some of the hematomas are detected on postoperative CT or MRI follow-ups and can be asymptomatic. The incidence of hemorrhages in different studies are varying. Fenoy et al [4] presented a retrospective summary of adverse events in 728 DBS patients treated by one neurosurgeon. In their study $3.4 \%$ had asymptomatic intraventricular hemorrhage, $1.1 \%$ presented symptomatic intracerebral hemorrhage and $0.5 \%$ had asymptomatic intracerebral hemorrhage. Several studies have looked into if intraoperative microelectrode recording (MER) increases the risk of hemorrhagic complications. Gorgulho et al [5] report from their study that $2.9 \%$ of patients undergoing MER presented with hemorrhage compared with $1.4 \%$ of patients undergoing only macro stimulation. Xiaowu et al [1] reported in their study of 644 patients that MER provides useful information for guiding surgery, but the procedure carries a risk of intracranial hemorrhage due to multiple electrodes and sharp tip of these microelectrodes. Other studies have not shown any increase in hemorrhagic complications using MER. Zibetti et al [6] describe a consecutive study of 221 patients with a total of 442 quadripolar electrodes implanted using MER, no association of increased risk of ICH using MER if the patient selection is careful and the surgical procedure is done by a well-trained team. Other patient safety intraoperative physiological methods for reaching the optimal target site, except from MER can be local field potentials from the target site using the macroelectrode, which records the neurophysiological activity and can help to identify anatomical borders [7]. Dynamic 
intraoperative impedance measurement is yet another method used by some surgeons [8]. The issue of proving the best possible safety for these patients undergoing the procedure is crucial, most of the described safety procedures like MER are for improving targeting, yet the most devastating complication is ICH. A considerable number of the patients are treated for hypertension which is a risk factor for ICH due to the vasosclerosis in the patients [9]. Patients with medicated hypertension should have well controlled blood pressure prior to surgery. Meticulous trajectory planning and optimal MRI sequences are some of the possible measures that can be done. For further decreasing the numbers of ICH and aiming for a zero ICH vision, it is also necessary to introduce intraoperative measurement techniques that can reduce the risk for hemorrhage and thus identify vessel structures along the trajectories. This is important not only for DBS-surgery but also for other types of stereotactic interventions such as radiofrequency lesioning and biopsy procedures.

One way already explored by us is the ability to in vivo monitor the microcirculation when creating the trajectory for the DBS lead by means of laser Doppler flowmetry (LDF) and a forward looking probe. Laser Doppler flowmetry is a well-explored method for monitoring skin and other tissue's microcirculation [10] and has previously been adapted to stereotactic neurosurgery $[11,12]$. An additional advantage with LDF is that it can also detect grey-white tissue boundaries in a similar way as reflectance spectroscopy performed in the near infrared wavelength interval [12-16]. A review of the steps in developing a tool for optical intraoperative guidance during stereotactic procedures is presented in [17]. In the present study a retrospective safety analysis using the optical navigation technique is presented for 83 lead implantations at the Department of Neurosurgery, Linköping University Hospital. The aim of the study was to investigate the applicability and safety of the LDF measurements during stereotactic DBS implantations.

\section{Material and Methods}

\section{Patients}

50 patients (18 female and 32 male) aged between 41 and 81 years (mean \pm sd, $65 \pm 9$ years) referred for uni- or bilateral DBS implantation at the Department of Neurosurgery, Linköping University Hospital were included in the study. In total 83 leads were implanted; 24 in ventral intermediate nucleus (VIM), 38 in the subthalamic nucleus (STN), 8 in the globus pallidum internus (GPi), 12 in the zona incerta (ZI) and one patient had a DBS lead implanted due to 
facial pain in the ventral posteromedial nucleus (VPM) of the thalamus. The studies were approved by the local ethics committee at the University Hospital in Linköping (D. no. M18204, T54-09) and informed written consent was received from the patients.

\section{Surgery}

The surgical procedures followed the protocol of an image guided frame-based approach to the STN, GPi and ZI targets. A stereotactic CT (slice thickness $1 \mathrm{~mm}$, GE Lightspeed Ultra, GE Healthcare, UK) was performed the day of surgery with the Leksell Stereotactic System ${ }^{\circledR}$ (LSS, Model G, Elekta Instrument AB, Sweden). The stereotactic CT was image fused with a preoperative MRI. Direct anatomical targeting of the STN, ZI and GPi was done on 1.5T or 3T MRI (T1 and T2, slice thickness $2 \mathrm{~mm}$, Philips Intera, The Netherlands). Indirect targeting of the VIM nucleus was performed and optimally the tip of the electrode was located on the AC-PC plane, 5-7 mm in front of PC with a laterality of 12-14 mm from midline. Targeting and planning of the trajectories were performed in the Leksell ${ }^{\circledR}$ Surgiplan System (Elekta Instrument AB, Sweden). Patients undergoing DBS implantation for STN, GPi and ZI-targets underwent the procedure in one stage in general anaesthesia. Patients with ET undergoing VIM surgery had their procedure done in two steps where the placement of the electrodes were done in local anaesthesia so the patients could be evaluated clinically with macro stimulation. Microelectrode recording was not performed in any of the cases in this review. The optical probe was used to create a trajectory and replaced the conventional guide used before. The trajectories were planned meticulously avoiding visible blood vessels, sulci and the lateral ventricles. All procedures were done by the same two surgeons.

\section{Postoperative imaging}

All 50 patients included from Linköping in the study during September 2005 to June 2016 were postoperatively examined with CT or MRI and co-registered with preoperative stereotactic MRI using Surgiplan software for confirmation of lead positions. The postoperative examination is since 2010 performed within $24 \mathrm{~h}$ from implantation and therefore these patients can be evaluated not only for lead positioning but also for any visible hemorrhage on the postoperative CT scan. Thirty-one patients in the study had the postoperative scan within $24 \mathrm{~h}$ and were evaluated for the presence of hemorrhage by a neurosurgeon and a neuroradiologist. 19 patients had their scan for evaluating lead positioning within a year from initial surgery but since there is no examination in the immediate postoperative period ( 24 - $48 \mathrm{~h}$ ) it cannot be excluded that there could have been 
small amounts of blood even though the patients showed no neurological symptoms from a suspected hemorrhage.

\section{Optical measurements}

System and probe

A LDF system (Periflux 5000, Perimed AB, Sweden) adapted and approved for clinical use at Linköping University Hospital during stereotactic neurosurgery was used for all the measurements. An in-house software in LabVIEW (National Instruments, Inc., TX, USA) made it possible to record both the brain tissue's microcirculation (also denoted blood flow or perfusion) and the backscattered total light intensity (TLI) which corresponds to the greyness of the tissue. The total measurement range of the perfusion and TLI signals are presented as 0-999 arbitrary units (a.u.) and 0-10 a.u. respectively. In the present LDF system, a TLI > 0.5 a.u. is required in order to process a perfusion signal. Low power laser light (wavelength 780 $\mathrm{nm}$, effect $1 \mathrm{~mW}$ ) is transmitted to the tissue from the LDF through an optical fiber positioned along the stainless steel shaft of the measurement probe. Receiving light is transmitted back to the LDF through the same probe for signal processing and presentation of the recorded result on-line in the operating room (OR). The probe's outer dimension was adapted to fit the LSS (length $190 \mathrm{~mm}$, diameter $2 \mathrm{~mm}$ except for the last $30 \mathrm{~mm}$ where it is $1.5 \mathrm{~mm}$ ), and with a forward looking smoothly rounded tip. Prior to each surgical procedure, the probe was tested in a calibration solution (Motility Solution, Perimed AB, Sweden) and if necessary the LDF recalibrated in order to make the measurements comparable, thereafter the probe was cleaned and sterilized using the STERRAD ${ }^{\circledR}$ procedure [18]. Figure 1 presents the measurement setup in the OR and Fig. 2 the probe as positioned in the LSS and the mechanical insertion device (Fig. 2a) and a sketch of the forward looking probe (Fig. 2b). A more detailed presentation of the system and the probe can be found in [12].

\section{Measurement procedures}

A similar measurement principle was used for all surgical procedures, but the insertion of the probe and measurement positions varied. In the first 8 trajectory recordings the probe was inserted manually by the surgeon. During these measurements the optical probe was carefully pushed down to $-30,-20,-10,-5,-2.5 \mathrm{~mm}$ from the pre-calculated target, and finally moved to the target region. For this set-up the perfusion and TLI signals were recorded during a total time period of $30 \mathrm{~s}$ per site [11]. In the remaining surgeries the mechanical hand-driven device was used to insert the probe in a millimeter precision controlled manner. As the initial 
measurements showed that the laser Doppler system presents a stable signal already within a few seconds after movement with the mechanical device from one position to another, the measurement time at each position could be reduced to about $10 \mathrm{~s}$. When the mechanical device was used, a recording was always done in the cortex, in white matter at the internal capsule (IC) as well as in the target region. For 22 implantations measurements were performed along the entire trajectory i.e. all the way from cortex towards the respective target with a distance between measurement positions of $1 \mathrm{~mm}$ [12]. For a second group of patients including 20 lead implantations a more detailed investigation of the microcirculation and tissue type was done starting $10 \mathrm{~mm}$ from the pre-calculated target and by using increments of $0.5 \mathrm{~mm}$ between measurement sites [19]. Along 23 of the trajectories, measurements were also performed 1-3 mm beyond the target. Additionally, the probe was used as guide to create the path for the leads simultaneously as LDF recordings in 33 patients.

\section{Data analysis}

All medical patient charts and postoperative radiology were retrospectively reviewed for hypertension, postoperative hemorrhage and DBS lead infections.

All measurement positions were reviewed from a microcirculatory perspective. As the microvascular blood flow in general is low (usually $<50$ a.u.), 50 a.u. was used as the threshold. Blood flow values being at least twice as high (> 100 a.u.) were considered as high blood flow spots. Furthermore, these were divided into the following group A: 101-250 a.u., B: 251-500 a.u. and C: > 500 a.u. according to previously presented analysis [12]. In addition, the spots with increased blood flow were compared with the TLI signals at the same site. At 52 sites the TLI was less than 0.5 a.u., it was not possible to process the perfusion signal because of the little reflected light. If such low TLI values appeared next to a high blood flow peaks they were set to the maximum perfusion value of 999 a.u. A typical LDF recording is shown in Fig. 3 and the A and B perfusion levels are marked with lines in order to exemplify high blood flow values.

\section{Results}

The retrospective safety analysis showed that all patients on systemic anticoagulation therapy, e.g. Warfarin had their medication discontinued 3-5 days before surgery. All of the patients received Tinzaparin sodium, a low molecular weight heparin the evening before surgery 
(10000 anti-Xa IE/ml, $0.25 \mathrm{ml}$ ) for thromboembolism prevention. The patients undergoing the procedure in general anesthesia $(n=35)$ received their anesthesia intravenously with Propofol and Remifentanil. Thirty-six of the patients had a history of hypertension and were on antihypertensive treatment at the time of surgery. All patients had normal blood pressure at time of surgery. Patients with borderline hypertension prior to surgery were referred back to their general practitioner for optimizing the antihypertensive treatment prior to surgery. No postoperative lead infections were detected.

Of the 2963 LDF measurement positions 234 (7.9\%) showed at least a doubled microvascular blood flow compared to the surrounding tissue. Of these measurements sites 131 (4.2\%) were A peaks. At 38 sites (1.3\%) the blood flow belonged to the B group and 65 (2.2\%) to the C group i.e. had more than five times higher blood flow in front of the probe tip. Of the Cvalues 52 were set to 999 a.u. The high blood flow peaks were most frequent in the cortex close to sulci, in the vicinity to the ventricles, and along VIM trajectories. All trajectories except one were recorded without adverse events. In one patient the stereotactic frame detached during surgery and the LDF system showed an atypical measurement. Due to this the surgery was inhibited. A postoperative CT scan revealed a small ICH along the trajectory as presented in Fig. 4 together with a mean curve of eleven optical trajectories towards the VIM (See also [12]). The patient was asymptomatic and admitted three months later for new DBS surgery. A left sided ventricular involvement in one patient is clearly visualized (Fig.5ab) as increased microvascular blood flow can be seen and a deeper dip in the TLI signal compared to the right hand side (Fig. 5a, c). The measurement time required for each trajectory was 7 to15 minutes depending on the number of positions and measurement time at each site.

\section{Discussion}

In this study 83 DBS lead implantations performed with an optical guide for creation of the trajectory have been reviewed from safety aspects. The study shows that this optical guided with a forward looking feature can help indicate regions with increased blood flow as well as distinguish between the tissue’s greyness.

Deep brain stimulation surgery and other functional neurosurgical procedures aim at improving quality of life in patients with progressive neurological diseases, e.g. PD. The aim is to reduce symptoms of the disease and it is therefore very important to reduce surgical risks 
that can cause further disability for the patients. In neurosurgery there is a constant development of intraoperative monitoring techniques. Well established monitoring techniques include neurophysiological monitoring in spinal and tumor surgery, intraoperative MRI in surgery of low grade gliomas and awake craniotomy. In DBS surgery the most widespread and frequently used intraoperative monitoring technique, except for the anaesthetized patient, is MER, for optimizing the implantation position for the lead. There are no techniques in clinical use for routinely measuring the microcirculation in vivo during the stereotactic surgical procedure.

The incidence of hemorrhage in DBS surgery is low but it can be the most devastating complication DBS surgery. Tonge et al. [20] report from a recent published study an overall calculated risk of an ICH to be $1.81 \%$ per patient, 0.94 per side. To identify patient risk factors, high quality MRI and meticulous planning of trajectories including avoiding brain sulci and ventricles are important steps to minimize the risk of hemorrhage [21]. Even with high quality MRI small vessels in front of the guiding tube or electrode cannot be visualized; therefore a method for online, in vivo measurement of the brain microcirculation deep in the brain in front of the guiding tube could further increase patient safety by reducing the incidence of hemorrhage. Laser Doppler flowmetry for measuring the microcirculation during stereotactic surgery is a safe method with no complications reported in our material and it is not especially time consuming. A recording along one lead is done within 7 to15 minutes, depending on the protocol. The mechanical device used and described is easy to handle for the surgeon and also the interpretation of the perfusion curves. The LDF technique can further decrease risks of hemorrhage in this already vulnerable group of patients. It can also be used for all targets in DBS surgery and for other stereotactic procedures like biopsies of deep brain tumors. New probe designs would also allow MER in combination with LDF. The LDF can be used for patients undergoing procedures both in general anesthesia or in local anesthesia.

The purpose of this study was to review the optical measurements performed in 50 patients. A weakness with the study is that the first 19 patients did not routinely undergo postop radiology within 24 hours. During the course of the study, however, the DBS implantation procedure has successively been improved including today routine postoperative imaging. Furthermore the entire procedure is done in one session and with general anesthesia in order to reduce the discomfort of the patient. The total time today is about 4 hours for a unilateral and 5 hours for a bilateral procedure including LDF measurements and pulse generator 
implantation. All recordings were done with the same type of forward looking probe, but with various measurement protocols and methodological improvements. During surgery the probe was used as a guide creating the trajectory for the DBS lead at the same time as the hyper perfused tissue in the vicinity to the probe tip was presented on-line in the OR together with grey-white tissue changes. At present time, the "bar-codes” for optical trajectories are processed postoperatively. An improvement in a future system would be to present both the blood flow peaks and "bar-codes" in real-time together with the preoperative images

\section{Conclusion}

The risk of developing ICH as presented in the literature varies, probably due to variations in preoperative assessment and surgical approaches and how different centers manage this problem. In this study 83 DBS lead implantations show that the probe with optical fibers does not increase the hemorrhage incident compared to other intraoperative measurement techniques. Instead the probe's forward looking feature can identify increased blood flow in the vicinity to potential vessel structures, and also acts as an intraoperative guidance method for distinction between shades of grey matter and white matter along the predefined trajectory. As the probe also acts as the guide and thus creates the trajectory for the DBS-lead, no separate guide tube is necessary.

\section{Acknowledgements}

Johan Richter, MD and the Staff the Department of Neurosurgery, Linköping University Hospital are acknowledged for skilful assistance during the measurements. The authors are also very grateful for all previous participants in the project who assisted in the optical measurements and the analysis. Please see authors list in refereed papers. The study was supported by the Swedish Research Council (621-2013-6078) and the Parkinson Foundation at Linköping University. Imaging was performed at Center for Medical Image Science and Visualization (CMIV). The authors do not have any conflict of interest to report. 


\section{Legends to Figure}

Figure 1. Overview of the setup in operating room using the optical measurement technique (a). Recording and analysis of blood flow parameters as a close up (b): microvascular blood flow is presented in yellow and total backscattered light corresponding to tissue greyness in blue. The DBS surgeon operating the mechanical insertion device during surgery (c).

Figure 2. The mechanical insertion device and optical probe mounted on the Leksell ${ }^{\circledR}$ Stereotactic System (a). The forward looking probe is illustrated in (b).

Figure 3. Example of recorded blood flow signal (perfusion in orange) and total light intensity (TLI in blue) $-41 \mathrm{~mm}$ to $-32 \mathrm{~mm}$ from calculated target. Intervals for increased blood flow (denoted A, B and C) are marked with dotted lines. An example of a C peak is seen at $36 \mathrm{~mm}$.

Figure 4. Postoperative CT scan (5mm slices) with a minimal asymptomatic intracerebral hemorrhage (ICH) along the trajectory marked in the middle panel (a). Optical curves presenting the microcirculation/perfusion (b) and the tissue grayness i.e. total light intensity (TLI) (c). The mean curve is based on data from ten trajectories towards the VIM nucleus. The deviating curves were due to the small ICH seen in (a). No perfusion signal could be recorded in the interval 32 to $22 \mathrm{~mm}$ due to little light reflected back, due to the bleeding in front of the probe.

Figure 5. CT image presenting the probe passing through the ventricle on the left side (a). The optical trajectories show the microcirculation (b) and total light intensity (c) on the left (L) and right (R) hand side respectively. It is clearly seen that the microcirculation increase in the vicinity to the ventricle and that the TLI signal decreases. 


\section{References}

1. Xiaowu, H., J. Xiufeng, Z. Xiaoping, H. Bin, W. Laixing, C. Yiqun, L. Jinchuan, J. Aiguo and L. Jianmin, Risks of intracranial hemorrhage in patients with Parkinson's disease receiving deep brain stimulation and ablation. Parkinsonism Relat Disord, 2010. 16(2): p. 96-100.

2. Elias, W.J., C.A. Sansur and R.C. Frysinger, Sulcal and ventricular trajectories in stereotactic surgery. J Neurosurg, 2009. 110(2): p. 201-7.

3. Zrinzo, L., T. Foltynie, P. Limousin and M.I. Hariz, Reducing hemorrhagic complications in functional neurosurgery: a large case series and systematic literature review. $\mathrm{J}$ Neurosurg, 2012. 116(1): p. 84-94.

4. Fenoy, A.J. and R.K. Simpson, Jr., Risks of common complications in deep brain stimulation surgery: management and avoidance. J Neurosurg, 2014. 120(1): p. 132-9.

5. Gorgulho, A., A.A. De Salles, L. Frighetto and E. Behnke, Incidence of hemorrhage associated with electrophysiological studies performed using macroelectrodes and microelectrodes in functional neurosurgery. J Neurosurg, 2005. 102(5): p. 888-96.

6. Zibetti, M., A. Romagnolo, E. Crobeddu, R. Fornaro, A. Merola, M.G. Rizzone, L. Lopiano and M. Lanotte, Does intraoperative microrecording really increase the risk of hemorrhagic complications in deep brain stimulation? Brain Stimul, 2014. 7(6): p. 911-2.

7. Telkes, I., N.F. Ince, I. Onaran and A. Abosch, Localization of subthalamic nucleus borders using macroelectrode local field potential recordings. Conf Proc IEEE Eng Med Biol Soc, 2014. 2014: p. 2621-4.

8. Zrinzo, L. and M. Hariz, Impedance recording in functional neurosurgery, in Textbook of stereotactic and functional neurosurgery, P.L. Gildenberg, A.M. Lozano, and R. Tasker, Editors. 2008.

9. Terao, T., H. Takahashi, F. Yokochi, M. Taniguchi, R. Okiyama and I. Hamada, Hemorrhagic complication of stereotactic surgery in patients with movement disorders. $\mathrm{J}$ Neurosurg, 2003. 98(6): p. 1241-6.

10. Strömberg, T., K. Wårdell, M. Larsson and S. Salerud, Laser Doppler monitoring and imaging techniques, in Biomedical Photonics Handbook, T. Vo-Dinh, Editor. 2014, CRC. p. 435-459.

11. Wårdell, K., P. Blomstedt, J. Richter, J. Antonsson, O. Eriksson, P. Zsigmond, A.T. Bergenheim and M.I. Hariz, Intracerebral microvascular measurements during deep brain 
stimulation implantation using laser Doppler perfusion monitoring. Stereotact Funct Neurosurg, 2007. 85(6): p. 279-86.

12. Wårdell, K., P. Zsigmond, J. Richter and S. Hemm, Relationship between laser Doppler signals and anatomy during deep brain stimulation electrode implantation toward the ventral intermediate nucleus and subthalamic nucleus. Neurosurgery, 2013. 72(2 Suppl Operative): p. ons127-40.

13. Giller, C.A., H.L. Liu, P. Gurnani, S. Victor, U. Yasdani and D.C. German, Validation of a near-infrared probe for detection of thin intracranial white matter structures. $\mathrm{J}$ Neurosurg, 2003. 98: p. 1299-1306.

14. Antonsson, J., O. Eriksson, P. Blomstedt, A.T. Bergenheim, I.H. M, J. Richter, P. Zsigmond and K. Wårdell, Diffuse reflectance spectroscopy measurements for tissue-type discrimination during deep brain stimulation. J Neural Eng, 2008. 5(2): p. 185-90.

15. Giller, C.A., H. Liu, D.C. German, D. Kashyap and R.B. Dewey, A stereotactic nearinfrared probe for localization during functional neurosurgical procedures: further experience. J Neurosurg, 2009. 110(2): p. 263-73.

16. Johansson, J.D., P. Blomstedt, N. Haj-Hosseini, A.T. Bergenheim, O. Eriksson and K. Wårdell, Combined Diffuse Light Reflectance and Electrical Impedance Measurements as a Navigation Aid in Deep Brain Surgery. Stereotact Funct Neurosurg, 2009. 87(2): p. 105113.

17. Wårdell, K., Optical Monitoring Techniques for Navigation during Stereotactic Neurosurgery. Sensors and Materials, 2016. 28(10): p. 1105-1116.

18. Jacobs, P., Sterrad 100S, Sterilisation system. Johnson \& Johnson, 2006.

19. Wårdell, K., S. Hemm-Ode, P. Rejmstad and P. Zsigmond, High-Resolution Laser Doppler Measurements of Microcirculation in the Deep Brain Structures: A Method for Potential Vessel Tracking. Stereotact Funct Neurosurg, 2016. 94(1): p. 1-9.

20. Tonge, M., L. Ackermans, E. Kocabicak, V. van Kranen-Mastenbroek, M. Kuijf, M. Oosterloo, P. Kubben and Y. Temel, A detailed analysis of intracerebral hemorrhages in DBS surgeries. Clin Neurol Neurosurg, 2015. 139: p. 183-7.

21. Zrinzo, L., A.L. van Hulzen, A.A. Gorgulho, P. Limousin, M.J. Staal, A.A. De Salles and M.I. Hariz, Avoiding the ventricle: a simple step to improve accuracy of anatomical targeting during deep brain stimulation. J Neurosurg, 2009. 110(6): p. 1283-90. 


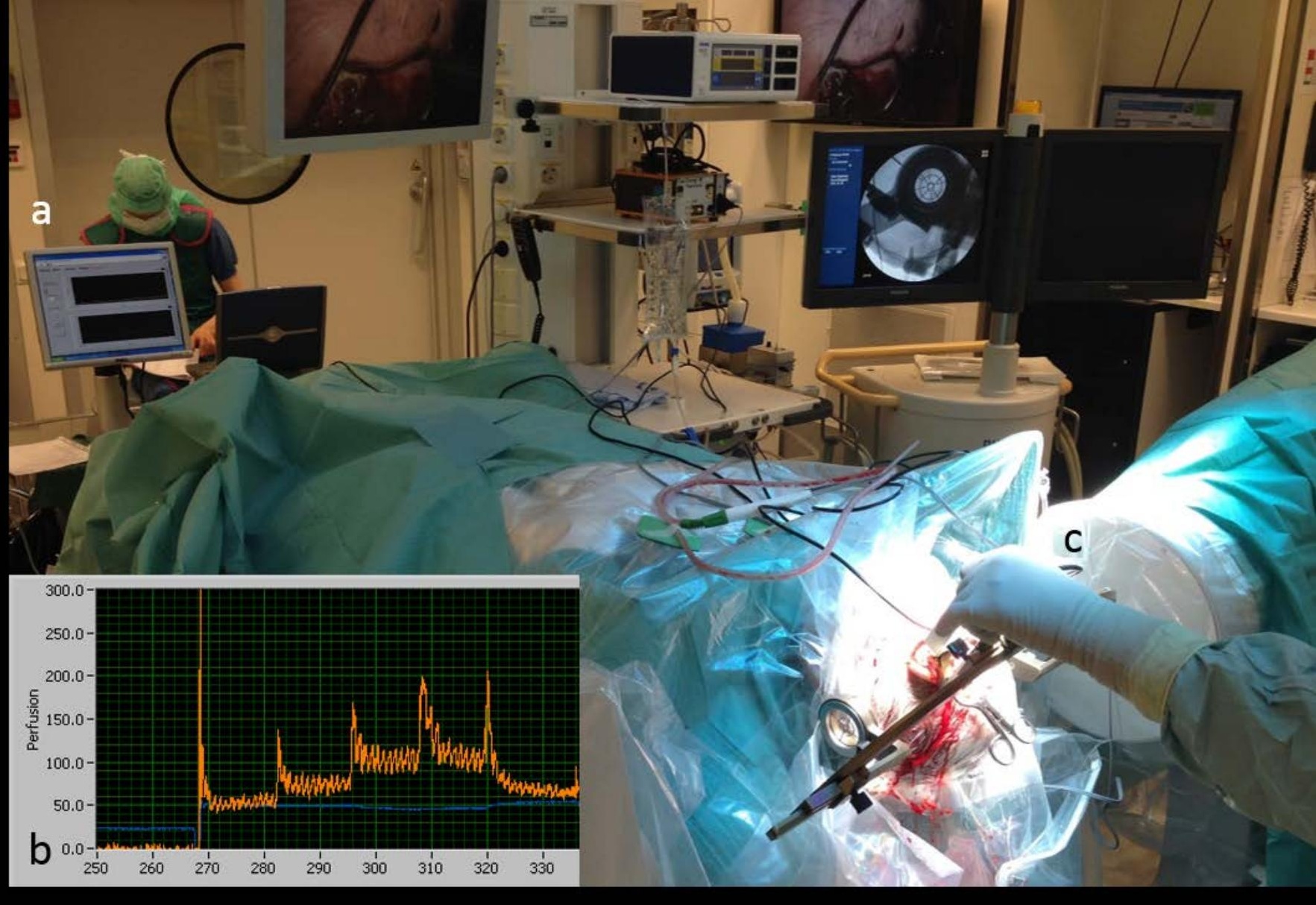




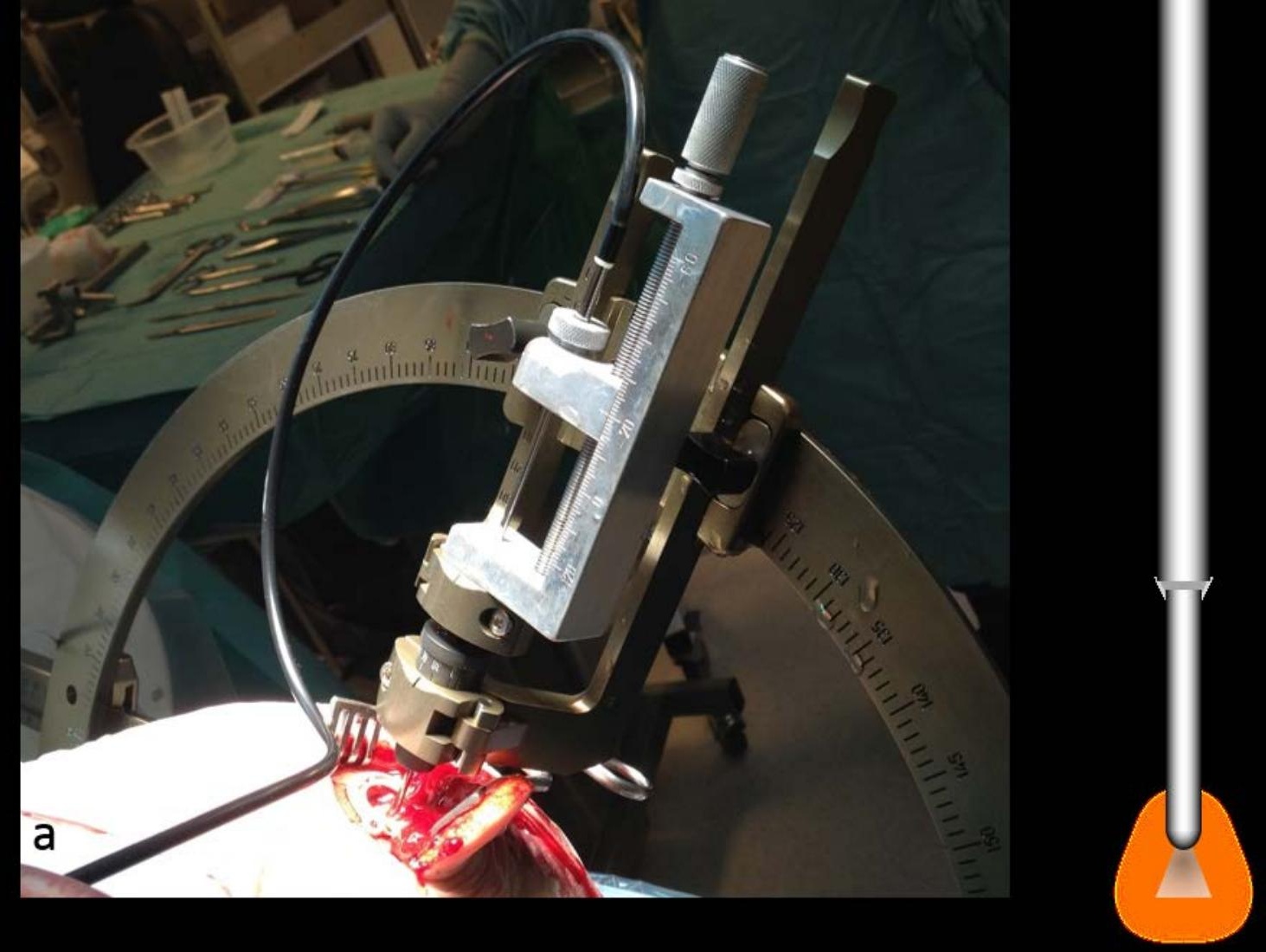




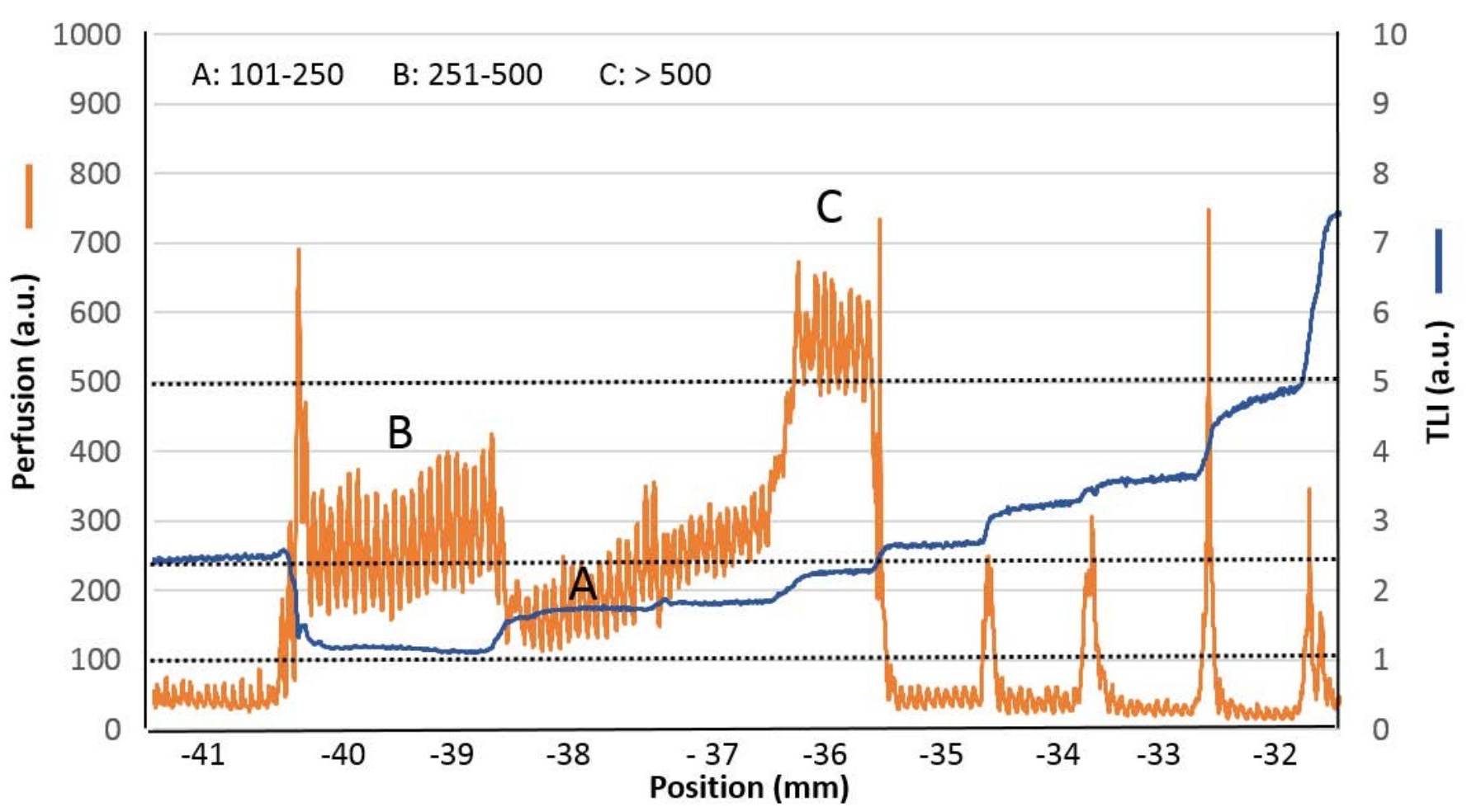



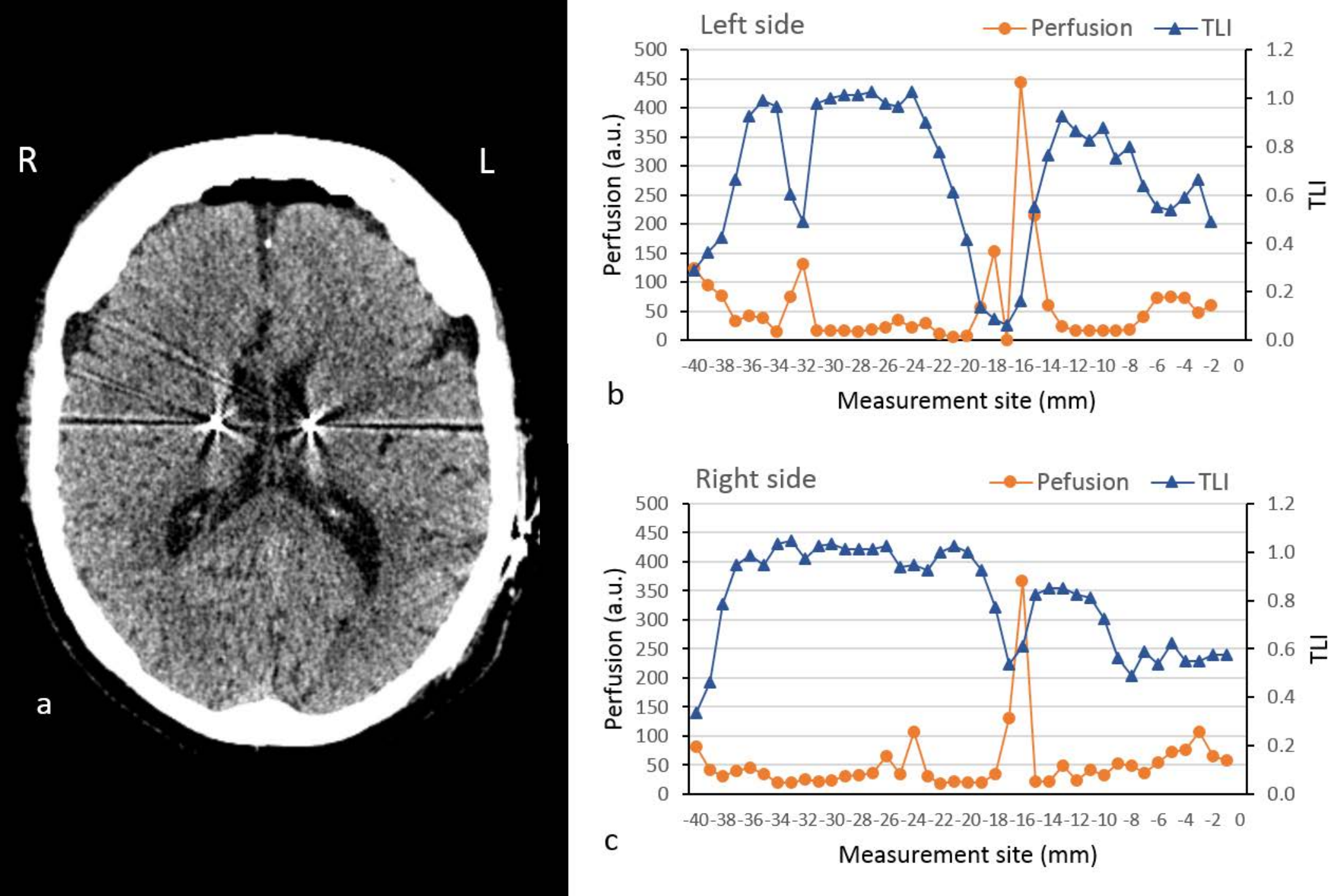